\title{
Complex Optimization and Simulation in Power Systems
}

\author{
João Soares $\mathbb{D}^{1},{ }^{1}$ Fernando Lezama $\mathbb{D}^{1},{ }^{2}$ Tiago Pinto $\mathbb{D}^{\mathbb{D}},{ }^{3}$ and Hugo Morais ${ }^{4}$ \\ ${ }^{1}$ GECAD, Research Group on Intelligent Engineering and Computing for Advanced Innovation and Development, \\ Polytechnic of Porto (IPP), R. Dr. António Bernardino de Almeida, 431, 4200-072 Porto, Portugal \\ ${ }^{2}$ National Institute of Astrophysics, Optics and Electronics (INAOE), Puebla, Mexico \\ ${ }^{3}$ BiSITE, University of Salamanca, Calle Espejo, 2, 37007 Salamanca, Spain \\ ${ }^{4}$ Électricité de France R\&D, Paris, France \\ Correspondence should be addressed to João Soares; jan@isep.ipp.pt
}

Received 6 September 2018; Accepted 6 September 2018; Published 14 October 2018

Copyright (C) 2018 João Soares et al. This is an open access article distributed under the Creative Commons Attribution License, which permits unrestricted use, distribution, and reproduction in any medium, provided the original work is properly cited.

Deregulation of energy markets has changed the way of delivering power to electricity customers while introducing serious competition [1]. Meanwhile, electricity demand has been increasing steadily, and the limited earth resources require more sustainability, which is critical to keep up the world at the current pace $[2,3]$. In this context, not only is energy efficiency crucial to contribute to sustainability, but also adequate methods of energy production and consumption are highly relevant (e.g., energy resource management) [4]. Optimization and simulation approaches are a key part of the planning, operation, and control of energy systems [5]. In addition to the ever-increasing penetration of renewables, the expected massive penetration of electric vehicles is challenging the power system and transportation industry as never before. Dealing with these new challenges while being able to create innovative and prosperous business ideas is essential for survival in the advent of smartening power systems. The complexity of optimization and simulation problems in this domain is increasing. Optimization is usually highly constrained and faces issues related to high-dimensionality, lack of information, and noisy and corrupted data as well as realtime requirements, while simulation requires multidomain knowledge (communications, market mechanisms, etc.) to capture the essence of the new paradigm of power systems.

This special issue (SI) entitled "Complex Optimization and Simulation in Power Systems" (COSPS) attracted several contributions in related topics with novel ideas to tackle complex problems in optimization and simulation in power systems. More precisely, COSPS SI attracted 33 submissions from researchers all over the world. From those submissions,
14 papers have been accepted, including a survey paper from the guest editors.

We accepted 13 out of the 33 received papers. Besides, our accepted survey paper reviews last decade work on the special issue topics entitled "Survey on Complex Optimization and Simulation for the New Paradigm of Power Systems". This survey paper was handled by external editors and reviewers. Presented papers in this special issue deal with complexity at different levels of the power system field. This special issue covers papers that deal with energy management algorithms at the bottom levels of the power system grid. Those papers explore the flexibility properties of demand, which has been considered crucial for the stability and sustained growth of future power systems (renewables and EVs integration [6]). In addition, this special issue provides an interesting discussion on the research of the most challenging problems in power systems, namely, economic dispatch and planning with advanced optimization algorithms such as Quantum Particle Swarm Optimization.

The paper "A Cosimulation Architecture for Power System, Communication, and Market in the Smart Grid" presents a cosimulation architecture for the complex representation of the new paradigm of power systems, namely, the smart grid. The multidomain simulation environment of power system, communication, and market mechanism is considered. Different requirements and use cases are identified and a comprehensive data model is proposed. A case study of virtual power plant is presented in the cosimulation framework and a communication network. 
The paper "Dealing with Demand in Electric Grids with an Adaptive Consumption Management Platform" explores flexibility in both homes and workplaces. A multiagent system with advanced reasoning and learning capabilities is proposed to tackle the problem and adjust consumption to the current demand situation automatically. The system seems to provide promising results.

The paper "An Evaluation of a Metaheuristic Artificial Immune System for Household Energy Optimization" proposes the use of artificial immune systems, which use special bioinspired algorithms, in the home energy management system. The work aims to present the application of an artificial immune system in the context of different energy optimization problems. Likewise, a case study is performed in which an artificial immune system is incorporated to solve an energy management problem in a domestic environment. A thorough analysis of the different strategies is carried out to demonstrate the ability of an artificial immune system to find a successful optimum which satisfies the problem constraints.

The paper "An Ising Spin-Based Model to Explore Efficient Flexibility in Distributed Power Systems" analyzes consumers' demand flexibility at distribution system level using an Ising spin-based model. A local exchange scheme is considered to enable energy trade. A modified MetropolisHasting algorithm is proposed to analyze the system on large scale, considering total aggregation. Results suggest that profit increases with the number of aggregators in a maximum flexibility scenario.

The paper "Optimal Scheduling of a Microgrid Including Pump Scheduling and Network Constraints" proposes an energy management system for industrial microgrids. The considered energy resources include diesel generators, battery energy storage systems, renewable energy sources, flexible loads, and interruptible loads. The network constraints are evaluated with an optimal power flow in a secondstage optimization. The results indicate that electricity costs are reduced thanks to adequate pump scheduling and load management strategies.

The paper "Analysis of Constraint-Handling in Metaheuristic Approaches for the Generation and Transmission Expansion Planning Problem with Renewable Energy" presents a multiperiod generation and transmission expansion planning problem. Using a stochastic approach, the authors can find expansion plans that satisfy the uncertainty conditions regarding demand and renewable generation.

The paper "An Elitist Transposon Quantum-Based Particle Swarm Optimization Algorithm for Economic Dispatch Problems" uses an advanced PSO to solve a highly nonlinear constrained economic dispatch problem. The authors compared their double elitist breeding quantum-based particle swarm optimization (DEB-QPSO) against state-ofthe-art algorithms, showing its superiority in different testbeds.

The paper "Minimizing Harmonic Distortion Impact at Distribution System with Considering Large-Scale EV Load Behaviour Using Modified Lightning Search Algorithm and Pareto-Fuzzy Approach" proposes a multiobjective lightning search algorithm for optimal placement sizing of variable passive filters to mitigate harmonic distortion due to charging stations of a large-scale EV fleet. The analysis carried out is modelled in MATLAB/m-file platform, using High Performance Computing (HPC) to make simulation efficient. Authors concluded that their approach is suitable to mitigate harmonic and minimize the impact of large-scale charging station installations.

The paper "Techno-Economic Distribution Networks Planning Using Smart Grid Techniques with Evolutionary Self-Healing Network States" proposes a new model tool for distribution network planning called scenario investment planning. The tool uses advanced computational resources to help network operators to visualize and design operational planning investments in the short and long terms.

The paper "Simulation Study on Clustering Approaches for Short-Term Electricity Forecasting" addresses the topics of load profiling and forecasting. A comprehensive study of clustering methods to identify residential electricity demand profiles and forecast energy consumption is provided. The paper explores the most relevant time series similarity measures, including measures based on the shape of time series, measures based on editing the time series, and measures based on the time series features. The paper also provides a discussion on measures for determining the relevant number of clusters, which comprehends fourteen alternative indexes. A case study using data from forty-six households in Austin, Texas, is performed, in which the similarity measures from different time series are analyzed. The experiments are conducted using nine different segmentation-enhanced forecasting algorithms. The paper concludes that of all the developed methods, neural network models, are the ones that can achieve the best results for aggregating the entire population for hourly data and that the forecasting error decreases when the number of clusters increases. Finally, the paper discusses the implications of the results from the operator's perspective.

The paper "A Novel Control Strategy on Multiple-Mode Application of Electric Vehicle in Distributed Photovoltaic Systems" addresses the integration of electric vehicles in the system. The authors propose a novel control scheme for electric vehicles with vehicle-to-grid capabilities. The proposed control structure enables the customer to achieve the lowest expense on electricity through the storage battery system control and electric vehicle optimal scheduling. The authors propose two day-ahead optimal control strategies with different objective functions, namely, minimizing the daily electricity expense of individual distributed PV system and minimizing the daily total expense of distributed PV systems to which vehicles can be connected to. The model is assessed using an entire year of historical data from 300 households with PV users, provided by a utility in Australia. The meteorological correlation parameters and electricity prices are also based on real data from Australia. Results show that the integration of electric vehicles with vehicle-to-grid capabilities as distributed storage can significantly increase the advantages for the system.

The paper "Investigation and Optimization of Grounding Grid Based on Lightning Response by Using ATP-EMTP and Genetic Algorithm" addresses an innovative method to determine the optimal grounding grid. In the paper, 
the transient methodology is introduced to investigate the lightning effect on grounding body at each point of grounding grid in normal and optimized conditions. Genetic algorithm is applied for regular and irregular grounding grid to obtain best values of mesh size with the lower ground potential rise (GPR) as compared with the normal condition for more safety. Several voltages on different positions of grounding grid are described in this paper using ATP-EMTP and genetic algorithm. The computer simulation presented in the paper allows concluding that the proposed scheme is highly feasible and technically attractive.

The paper "Bidirectional Tracking Robust Controls for a DC/DC Buck Converter-DC Motor System" proposes two new designs of differential flatness-based bidirectional tracking robust controls for a DC/DC Buck converter-DC motor system. The first design considers the complete dynamics of the system; i.e., it considers the DC/DC Buck converterinverter-DC motor connection as a whole. The second separates the dynamics of the Buck converter from the one of the inverter-DC motor, so that a hierarchical controller is generated. The experimental implementation of both controls was performed via MATLAB-Simulink and a DS1104 board in a built prototype of the DC/DC Buck converter-inverter-DC motor connection. Controls show a good performance even when system parameters are subjected to abrupt uncertainties. The paper concludes that the robustness of such controls is verified.

Having said this, we invite the reader to continue with us in this special issue throughout one or more of the 14 articles we briefly described above. The research proposals covered by this special issue indicate several directions which future work may tackle in order to deal with increasing levels of complexity in this field, namely, at optimization and simulation. Many times both coexist together and cannot be conceived, developed, and implemented separately.

\section{Conflicts of Interest}

The authors declare that there are no conflicts of interests regarding the publication of this paper.

\section{Acknowledgments}

We want to thank coauthors that contributed to our special issue COSPS by submitting their valuable works. We also thank the reviewers that worked voluntarily to produce high quality feedback of the submitted manuscripts. Thanks are also due to the remaining staff of Complexity who help us to reach this result. The present special issue has been possible to funding in part under the EUREKA-ITEA2 Project M2MGrids (ITEA-13011) and Project SIMOCE (ANI|P2020 17690) and FEDER funds through COMPETE program and from national funds through Fundação para a Ciência e a Tecnologia under the project UID/EEA/00760/2013. In addition, it was supported in part by Grant agreement no. 703689 (project ADAPT). Additionally, this work was partially supported by $02 /$ SAICT/2017, POCI-01-0145-FEDER028983 (project CENEGERTIC).

\author{
João Soares \\ Fernando Lezama \\ Tiago Pinto \\ Hugo Morais
}

\section{References}

[1] F. Lezama, J. Soares, P. Hernandez-Leal, M. Kaisers, T. Pinto, and Z. M. Almeida do Vale, "Local energy markets: paving the path towards fully transactive energy systems," IEEE Transactions on Power Systems, pp. 1-1, 2018.

[2] P. Siano, "Demand response and smart grids-a survey," Renewable \& Sustainable Energy Reviews, vol. 30, pp. 461-478, 2014.

[3] S. D. Ramchurn, P. Vytelingum, A. Rogers, and N. R. Jennings, "Putting the 'smarts' into the smart grid: A grand challenge for artificial intelligence," Communications of the ACM, vol. 55, no. 4, pp. 86-97, 2012.

[4] J. Soares, B. Canizes, M. A. Fotouhi Gazvhini, Z. Vale, and G. K. Venayagamoorthy, "Two-stage Stochastic Model using Benders' Decomposition for Large-scale Energy Resources Management in Smart grids," IEEE Transactions on Industry Applications, pp. $1-1,2017$.

[5] J. L. Bernal-Agustín and R. Dufo-López, "Simulation and optimization of stand-alone hybrid renewable energy systems," Renewable \& Sustainable Energy Reviews, vol. 13, no. 8, pp. 21112118, 2009.

[6] P. Sterchele, A. Palzer, and H.-M. Henning, "Electrify everything?" IEEE Power and Energy Magazine, vol. 16, no. 4, pp. $24-$ 33, 2018. 


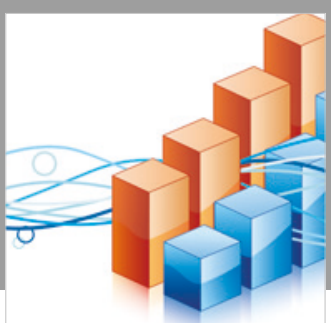

Advances in

Operations Research

\section{-n-m}
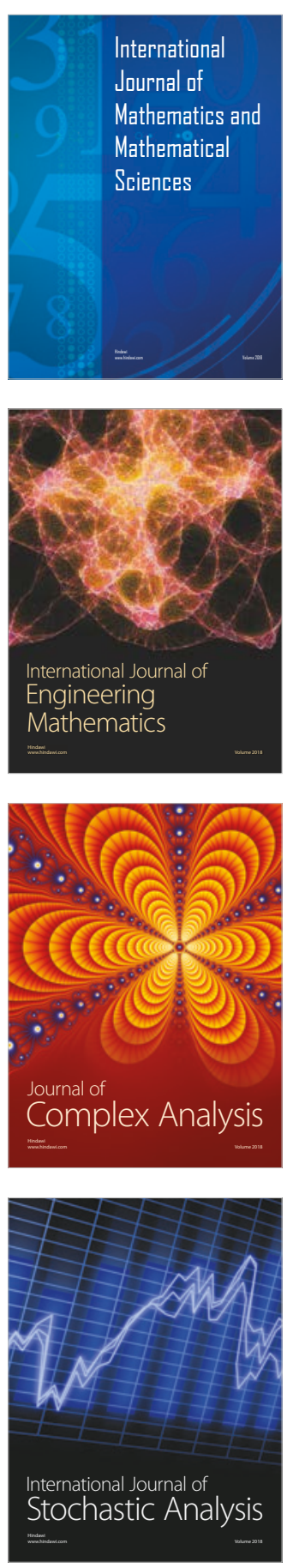
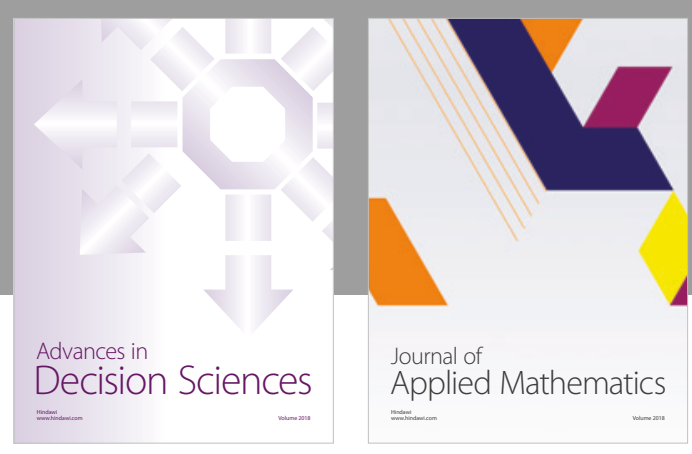

Journal of

Applied Mathematics
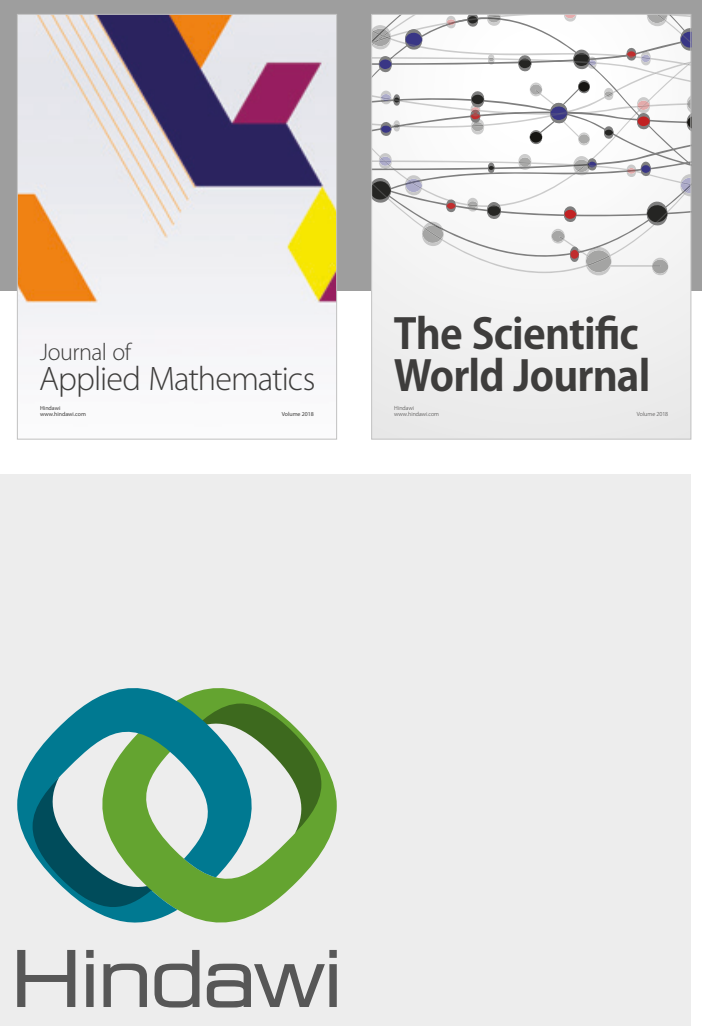

Submit your manuscripts at

www.hindawi.com

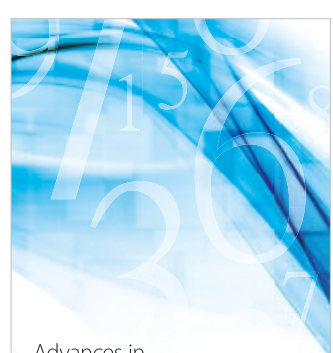

Advances in
Numerical Analysis
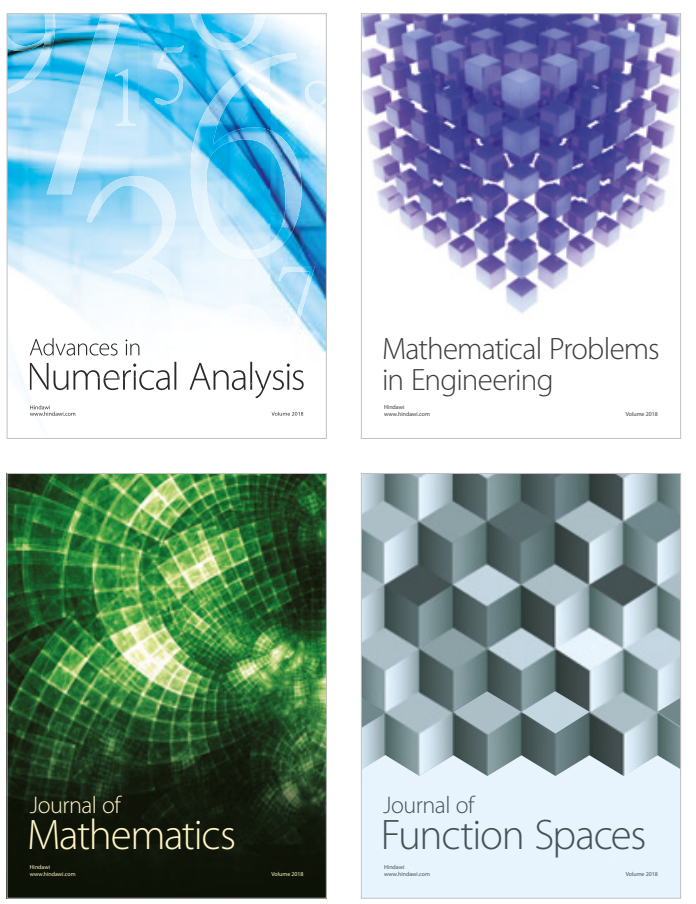

Mathematical Problems in Engineering

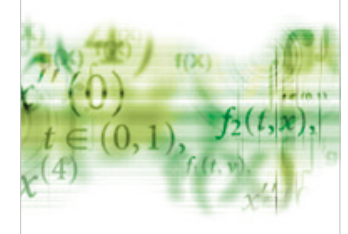

International Journal of

Differential Equations

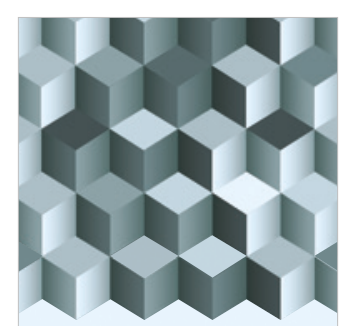

Journal of

Function Spaces
The Scientific

World Journal

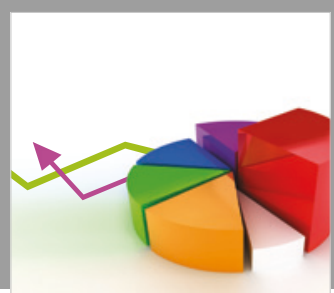

Journal of

Probability and Statistics
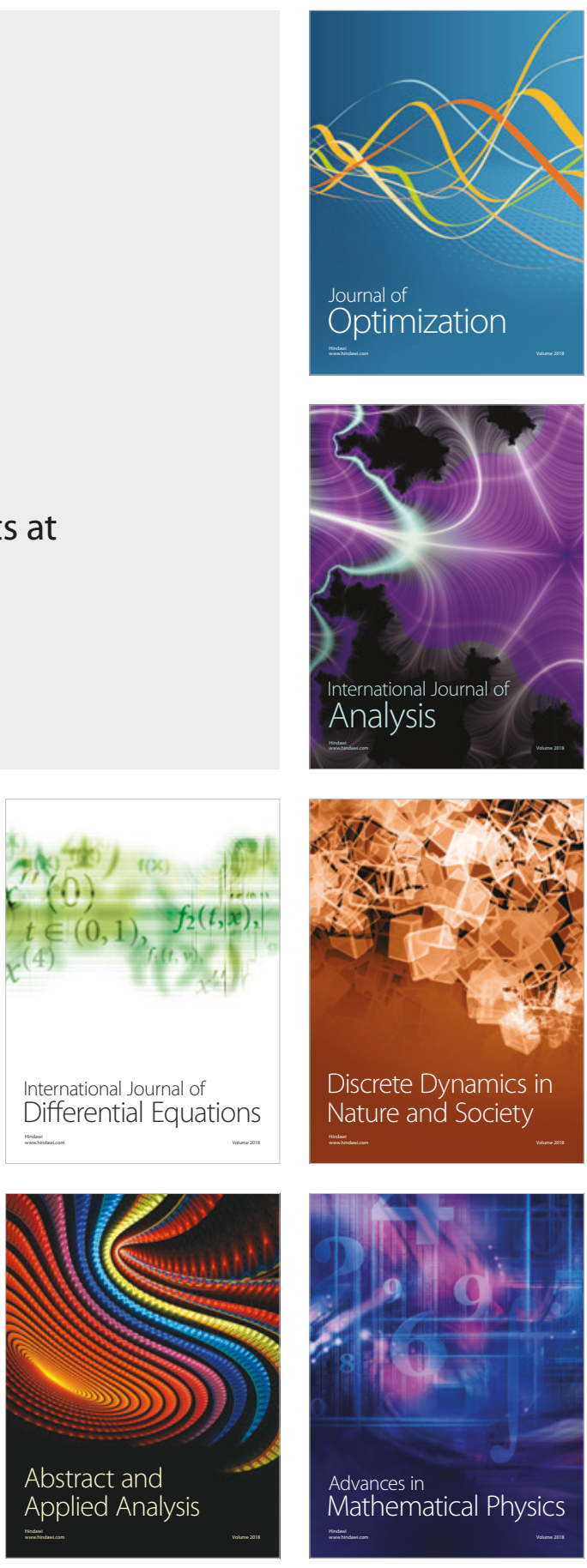